\title{
Platelet Parameters, C-Reactive Protein, and Depression: An Association Study
}

\author{
Jin-Min Wang ${ }^{1, *}$ \\ Kan-Di Yang ${ }^{2, *}$ \\ Song-Ying $\mathrm{Wu}^{3}$ \\ Xiang-Gu Zou ${ }^{3}$ \\ Yuan-Sheng Liao' \\ Bin Yang \\ Bu-Ni Xie' \\ Yan Huang ${ }^{4}$ \\ Shi-Ju Li \\ Hui-Jun $\mathrm{Ma}^{5}$ \\ 'Department of Internal Neurology, The \\ Second People's Hospital Affiliated to \\ Fujian University of Traditional Chinese \\ Medicine, Fuzhou, 350003, Fujian, \\ People's Republic of China; ${ }^{2}$ Department \\ of Internal Neurology, Anxi County \\ Hospital, Quanzhou, Fujian, 362400, \\ People's Republic of China; ${ }^{3}$ Institute of \\ Clinical Research, Fujian Academy of \\ Chinese Medical Sciences, Fuzhou, \\ 350003, Fujian, People's Republic of \\ China; ${ }^{4}$ Department of Internal \\ Neurology, Fuzhou Traditional Chinese \\ Medicine Hospital, Fuzhou, 35000I, \\ Fujian, People's Republic of China; \\ ${ }^{5}$ Department of General, Qiaokou Han \\ jia Dun Street Community Health Service \\ Center, Wuhan, 430000, Hubei, People's \\ Republic of China
}

*These authors contributed equally to this work
Objective: This study aims to investigate the correlation of platelet parameters and C-reactive protein (CRP) with depression.

Methods: The clinical data of 61 patients with depression and 30 healthy control subjects were collected to compare the platelet parameters, CRP levels, and Hamilton Depression Rating Scale (HAMD) scores of the two groups for correlation analysis.

Results: The results revealed that the body mass index (BMI) of patients with depression was lower $(\mathrm{P}<0.05)$ than that of the healthy control subjects, and that this difference was more significant in women than in men. Patients with severe depression showed an increased mean platelet volume (MPV) $(\mathrm{P}<0.05)$. In the patients with depression, MPV was positively correlated $(\mathrm{P}<0.05)$ with HAMD scores for work and interest, gastrointestinal symptoms, hopelessness, the anxiety/somatization factor, and the hopelessness factor. Platelet count (PLT) was negatively correlated $(\mathrm{P}<0.05)$ with HAMD scores for hypochondriasis, and plateletcrit $(\mathrm{PCT})$ was negatively correlated $(\mathrm{P}<0.05)$ with HAMD scores for middle insomnia and hypochondriasis. Platelet distribution width (PDW) was positively correlated $(\mathrm{P}<0.05)$ with HAMD scores for gastrointestinal and systemic symptoms as well as hopelessness. Higher CRP levels $(\mathrm{P}<0.05)$ were found in the patients with depression than in the healthy control subjects. Furthermore, in the patients with depression, CRP levels were positively correlated $(\mathrm{P}<0.05)$ with HAMD scores for guilt and the cognitive impairment factor.

Conclusion: Classical platelet parameters (PLT, MPV, PCT, PDW) and CRP were shown to be associated with specific depressive symptoms and cognitive impairment factors, including sleep, gastrointestinal symptoms, hypochondriasis, losing interest in work, and despair. These results suggest that both platelet parameters and CRP could be suitable biomarkers for predicting the occurrence and prognosis of depression, thus providing a new target for its treatment.

Keywords: depression, platelet parameters, C-reactive protein, Hamilton Depression Rating Scale

\section{Introduction}

Depression is a common mental disorder. Its core symptoms include persistent black moods, loss of interest in activities of daily living, and persistent fatigue. It was predicted that, by 2020, depression would become the second most prevalent type of disease worldwide, after cardiovascular and cerebrovascular diseases. ${ }^{1}$ Inflammation is likely a critical disease modifier, promoting susceptibility to depression. Controlling inflammation might provide an overall therapeutic benefit, regardless of whether it is secondary to early life trauma, a more acute stress response, microbiome alterations, genetic diathesis, or a combination of these and
Correspondence: Jin-Min Wang Department of Internal Neurology, The Second People's Hospital Affiliated to Fujian University of Traditional Chinese Medicine, No. 282 of Wusi Road, Gulou District, Fuzhou, 350003, Fujian, People's Republic of China

Tel +86-13960769905

Fax +86-59l-87825344

Email drwang_wjmI298@I63.com 
other factors. ${ }^{2}$ A large retrospective study found that students who reported depression showed an increased likelihood of exhibiting ear infections, bronchitis, sinus infections, and strep throat. ${ }^{3}$ There is also evidence that various bacterial and viral infections are associated with a range of depressive symptoms. ${ }^{4}$

Platelets are increasingly considered a bridge between mental, immunological, and coagulation-related disorders. The standard parameters associated with platelets include platelet count (PLT), mean platelet volume (MPV), plateletcrit (PCT), and platelet distribution width (PDW). These parameters also reflect platelet generation, which has a relationship with depression. Studies have shown that MPV is increased in patients with depression as a result of increased sympathetic nerve cortisol secretion and higher catecholamine levels, as well as adrenaline activation of adrenal receptors. ${ }^{5}$ Research in a Chinese population revealed that both MPV and PLT levels decreased in 31 female inpatients after antidepressant treatment. ${ }^{6}$ Both clinical and experimental studies have proposed that traditional drugs for inhibiting platelet aggregation, such as aspirin, can enhance the treatment effects of antidepressant medications when used in combination, and can also have an antidepressant treatment effect when used alone. ${ }^{7,8}$

Researchers have also investigated the relationship between major depressive disorder (MDD) and platelets by testing the platelet parameters. A study in an adult Turkish population sample revealed a significant independent association between MDD and MPV levels, with MPV being significantly higher in individuals with MDD. ${ }^{9}$ Studies have also reported significantly decreased MPV and PDW levels in patients with mild cognitive impairment and Alzheimer's disease (AD) compared with control subjects, reducing further as cognitive level declined, ${ }^{10,11}$ while other research has found reduced levels of PDW but increased levels of MPV in AD patients. ${ }^{12}$ The parameter of PDW, an index expressing heterogeneity of platelet size, has been shown to be negatively associated with psychological resilience and positively correlated with depressive symptoms. ${ }^{13}$ As such, PDW might be a useful marker of platelet function in developing a new potential biomarker of depression and conditions in the neuropsychiatric domain. ${ }^{14}$

C-reactive protein (CRP) is an acute-phase protein, and it is the most commonly used marker of systemic inflammation in humans. It is mainly synthesized and degraded by the liver, responding to the inflammatory state of the body. It is probable that the release of CRP and inflammatory cytokines leads to norepinephrine and 5-HT system dysfunction, thus causing depressive symptoms. ${ }^{15}$ Miler et $\mathrm{al}^{16}$ showed that CRP in the plasma was increased in patients with depression, ${ }^{17}$ and a meta-analysis study also found higher levels of CRP in the blood in patients with MDD than in a normal control population. ${ }^{18}$ These studies both demonstrated abnormally elevated CRP levels in patients with depression. In addition, CRP is associated with higher suicidality in patients with mental disorders, further supporting that there is a close correlation between depression and CRP. ${ }^{2}$

However, the correlations of PLT parameters and CRP with depression remain unclear due to the associations between CRP levels, platelet parameters, and specific depressive symptoms being relatively poorly studied. Therefore, the present study aims to investigate further the correlations of PLT parameters and CRP with depression to provide valuable clues for the clinical diagnosis and treatment of depression.

\section{Materials and Methods Object of the Study}

In the present study, patients with depression who were treated in our hospital between March 2017 and February 2019 were included in the depression group, while healthy subjects who participated in a physical examination were included in the control group. Diagnosis of depression for this research was performed in accordance with the diagnostic criteria in the International Classification of Diseases, 10th Revision, requiring at least two items of typical symptoms and other symptoms. The diagnosis for more severe forms of depression requires that the symptoms should last for two weeks or more. The typical depressive symptoms include (1) a black mood, (2) loss of interest and pleasure, and (3) fatigue. Other symptoms include (1) a decrease in the ability to focus, (2) a decrease in self-evaluation and selfconfidence, (3) a feeling of self-guilt and lack of a sense of value (also observed in mild depression), (4) a dim and pessimistic understanding of the future, (5) thoughts or behaviors of self-injury or suicide, (6) sleep disorder, and (7) alteration of appetite. ${ }^{2}$

The present study was performed in accordance with the Helsinki Declaration of the World Medical Association and was approved by the Ethics Committee of The Second People's Hospital Affiliated to Fujian University of 
Traditional Chinese Medicine. All participants provided signed informed consent.

\section{Inclusion and Exclusion Criteria}

The inclusion criteria for the depression group were as follows: (1) the patient's place of residence was located in Fujian province; (2) the patient was 18-60 years old; (3) the patient met the above criteria for the Western medicine diagnosis of depression; (4) the patient was aware and accepted undertaking the Hamilton Depression Rating Scale (HAMD) scale survey and assessment; (5) the patient's symptoms recurred after stopping drugs for more than two months or without any antidepressive treatment; and (6) the patient voluntarily participated in the clinical trial and provided signed informed consent.

The inclusion criteria for the control group were as follows: (1) the subject's place of residence was located in Fujian province; (2) the subject was 18-60 years old; (3) the subject was able to communicate normally and had no severe language or hearing impairment; (4) the subject had a HAMD score of $<8$ out of 24 items; and (4) the subject did not have any mental disorder such as mania or dissociative identity disorder.

The exclusion criteria were as follows: (1) patients $>60$ or $<18$ years old; (2) women who were pregnant or lactating; (3) patients who were addicted to or reliant on alcohol or drugs; (4) patients with severe heart, brain, or kidney diseases; (5) patients with awareness disorders, aphasia or agnosia, preventing them from communicating; (6) patients suffering from hyperthyroidism, autoimmune diseases, or cancer; (7) patients with a history of hypertension, hyperlipidemia, myocardial infarction, coronary atherosclerotic heart disease, or cerebral infarction; (8) patients suffering from hematological diseases; (9) patients with infectious diseases, eg, abscesses, pneumonia, or chronic obstructive pulmonary disease; (10) patients who had received hormonal drugs or contraceptives within the previous six months; and (11) patients who had received drugs for inhibiting platelet aggregation or antiinflammatory drugs within the previous three months.

\section{Participant Loss and Removal Criteria}

The data of subjects who refused to undertake the relevant examinations and tests were excluded from the study.

\section{Research Method}

Before enrollment in the study and taking the relevant medicine, each patient underwent a HAMD assessment (scores:
$<8=$ no depression; $8-20=$ mild depression; $21-35=$ moderate depression; $>35=$ severe depression) on the day before blood sample collection, performed by a full-time staff member. The general data for the included subjects and their results for the above scale were recorded in the case file. On the following day, elbow vein blood was collected under fasting status. Then, EDTA-K2 anticoagulant was added, and the sample was fully shaken. Within two hours of sample collection, routine blood tests were performed using the resistivity and fluorescence methods, a SYSMEX XN-3000 blood cell analyzer, and the supporting diluent. For CRP testing, the whole blood sample was measured by latex-enhanced immunoturbidimetry (BC5390, Mindray, China), and the coefficient of variation of the CRP measurements was $<8.3 \%$.

\section{Statistical Method}

All data were expressed as mean $\pm \mathrm{SD}$ or median (P25, P75). One-way analysis of variance was used to compare the differences in MPV levels between the different groups. The Mann-Whitney $U$-test was used to compare the differences between PLT, PCT, PDW, and CRP levels of the different groups. Spearman correlation analysis was used to analyze the correlations of HAMD items with platelet parameters and CRP levels. All statistical analysis was performed using SPSS v20.0, and a $\mathrm{P}$ value of $<0.05$ was considered statistically significant.

\section{Results \\ Comparison of Demographic Characteristics Between Two Groups}

There were 19 male and 42 female patients in the depression group. The mean age of these patients was $41.98 \pm$ 10.78 years, and the mean body mass index (BMI) was $21.25 \pm 3.18$. There were six male and 24 female patients in the control group. The mean age of these patients was $37.10 \pm 12.86$ years, and the mean BMI was $23.01 \pm 3.68$. The analysis results revealed that there was no statistically significant difference in gender or age $(P>0.05)$; however, BMI was significantly lower in the depression group than in the control group $(\mathrm{P}<0.05$; Table 1$)$.

\section{Comparison of Platelet Parameters in Patients with Different Levels of Depression}

The 61 patients with depression were divided into three groups according to their HAMD scores: a mild depression group, a moderate depression group, and a severe depression 
Table I Comparison of Demographic Characteristics Between the Two Groups

\begin{tabular}{|c|c|c|c|}
\hline & $\begin{array}{c}\text { Depressed } \\
(n=61)\end{array}$ & $\begin{array}{c}\text { Controls } \\
(n=30)\end{array}$ & $P$ value \\
\hline \multicolumn{4}{|l|}{ Gender } \\
\hline Male & 19 (31.1\%) & $6(20.0 \%)$ & 0.263 \\
\hline Female & $42(68.9 \%)$ & $24(80.0 \%)$ & \\
\hline Age $(Y)$ & $44(32.5,49.5)$ & $28(26,50)$ & 0.075 \\
\hline BMI $\left(\mathrm{kg} / \mathrm{m}^{2}\right)$ & $21.3(19.1,22.8)$ & $22.7(19.8,25.2)$ & $0.043^{*}$ \\
\hline Employed & 25 (4I.0\%) & 7 (24.1\%) & 0.119 \\
\hline Unemployed & $36(59.0 \%)$ & $22(75.9 \%)$ & \\
\hline \multicolumn{4}{|l|}{ Smoking } \\
\hline Current smokers & 2 (3.3\%) & I (3.4\%) & 0.967 \\
\hline Never smoking & 59 (96.7\%) & 28 (96.6\%) & \\
\hline \multicolumn{4}{|l|}{ Drinking } \\
\hline Current drinking & $6(9.8 \%)$ & $0(0.0 \%)$ & 0.195 \\
\hline Never drinking & 55 (90.2\%) & 29 (100.0\%) & \\
\hline \multicolumn{4}{|l|}{$\begin{array}{l}\text { Course of disease } \\
\text { (month) }\end{array}$} \\
\hline$<6$ & 30 (49.2\%) & 0 & - - - \\
\hline $6-12$ & $10(16.4 \%)$ & 0 & \\
\hline$>12$ & 21 (34.4\%) & 0 & \\
\hline
\end{tabular}

Note: ${ }^{*} \mathrm{P}<0.05$.

Abbreviation: BMI, body mass index.

group. The statistical results revealed statistically significant differences in MPV between the four groups $(\mathrm{P}<0.05$; Table 2; Supplementary Figure 1). However, there were no statistically significant differences between the PLT, PCT, or PDW values of the four groups ( $P>0.05$; Table 2$)$.

\section{Correlation Analysis Between Platelet Parameters and HAMD Scores in the Depression Group}

In the depression group, PLT was negatively correlated with the HAMD score for hypochondriasis, with a correlation coefficient of $-0.290(\mathrm{P}<0.05$; Table 3$)$, and PCT was negatively correlated with the HAMD scores for light sleep and hypochondriasis, with correlation coefficients of -0.276 and -0.279 , respectively $(\mathrm{P}<0.05$; Table 3 ). Meanwhile, PDW was positively correlated with the HAMD scores for gastrointestinal symptoms, constitutional symptoms, and hopelessness, with correlation coefficients of $0.255,0.272$, and 0.240 , respectively $(\mathrm{P}<0.05$; Table 3$)$, and negatively correlated with the HAMD score for hypochondriasis, with a correlation coefficient of $-0.240(\mathrm{P}<0.01$; Table 3$)$. In this group, MPV was positively correlated with the HAMD scores for work and interest, gastrointestinal symptoms, and hopelessness, with correlation coefficients of $0.275,0.251$, and 0.378 , respectively $(\mathrm{P}<0.05)$. Furthermore, MPV was positively correlated with the HAMD scores for the anxiety/ somatization and hopelessness factors, with correlation coefficients of 0.259 and 0.321 , respectively $(\mathrm{P}<0.05$; Table 4$)$.

\section{Comparison of C-Reactive Protein Between the Two Groups}

The CRP level of the patients in the depression group was $2.50(2.50,3.74) \mathrm{mg} / \mathrm{L}$, while that of the control group was $2.50(2.50,2.50) \mathrm{mg} / \mathrm{L}$. These results show that the CRP level of the patients in the depression group was higher than that in the control group, and the difference was statistically significant $(\mathrm{Z}=-3.495, \mathrm{P}<0.05$; Table 5; Supplementary Figure 2).

\section{Correlation Analysis Between C-Reactive Protein and HAMD Scores in the Depression Group}

The CRP level of the patients with depression was positively correlated with the HAMD score for guilt, and the correlation coefficient was $0.340, \mathrm{P}=0.007(\mathrm{P}<0.01$; Table 6). Furthermore, there was a positive correlation between CRP and the HAMD factor scores for cognitive

Table 2 Comparison of Platelet Parameters in Patients with Different Levels of Depression

\begin{tabular}{|l|c|c|c|c|c|}
\hline & $\mathbf{n}$ & PLT $\left(\times 1 \mathbf{0}^{\mathbf{9}} / \mathbf{L}\right)$ & MPV (fL) & PCT (\%) & PDW (\%) \\
\hline Non-depression group & 30 & $245.00(224.50,283.50)$ & $9.83 \pm 0.93$ & $0.23(0.22,0.27)$ & $11.05(9.88,13.73)$ \\
Mild depression group & 20 & $239.00(191.50,308.25)$ & $9.77 \pm 1.20$ & $0.25(0.19,0.28)$ & $11.80(9.63,13.60)$ \\
Moderate depression group & 28 & $252.50(217.50,273.00)$ & $9.83 \pm 0.73$ & $0.24(0.21,0.29)$ & $10.76(9.93,11.78)$ \\
Severe depression group & 13 & $210.00(184.50,269.00)$ & $10.15 \pm 0.91$ & $0.23(0.22,0.28)$ & $12.30(10.90,14.10)$ \\
$P$ & $-\cdots$ & 0.235 & $0.023 *$ & 0.980 & 0.142 \\
\hline
\end{tabular}

Note: $* P<0.05$

Abbreviations: PLT, platelet; MPV, mean platelet volume; PCT, plateletcrit; PDW, platelet distribution width. 
Table 3 The Correlation Analysis Between Platelet Parameters and HAMD Factor Score in the Depression Group

\begin{tabular}{|c|c|c|c|c|c|c|c|c|}
\hline \multirow[t]{2}{*}{ HAMD } & \multicolumn{2}{|c|}{ MPV } & \multicolumn{2}{|c|}{ PLT } & \multicolumn{2}{|c|}{ PCT } & \multicolumn{2}{|c|}{ PDW } \\
\hline & $\mathbf{r}$ & $P$ & $\mathbf{r}$ & $P$ & $\mathbf{r}$ & $P$ & $\mathbf{r}$ & $P$ \\
\hline Depressed mood & 0.041 & $0.75 I$ & 0.051 & 0.632 & 0.197 & 0.128 & 0.082 & 0.528 \\
\hline Guilty & 0.007 & 0.958 & -0.036 & 0.735 & 0.004 & 0.979 & 0.037 & 0.779 \\
\hline Suicide & 0.140 & 0.283 & 0.089 & 0.401 & 0.153 & 0.239 & 0.106 & 0.418 \\
\hline Difficulty falling asleep & 0.059 & 0.651 & 0.207 & 0.109 & -0.277 & 0.162 & -0.021 & 0.870 \\
\hline Light sleep & 0.037 & 0.779 & -0.199 & 0.059 & -0.276 & $0.031 *$ & -0.078 & 0.550 \\
\hline Early awakening & 0.048 & 0.714 & -0.031 & 0.765 & 0.079 & 0.543 & 0.060 & 0.649 \\
\hline Work and interest & 0.275 & $0.032 *$ & -0.030 & 0.775 & 0.089 & 0.497 & 0.087 & 0.507 \\
\hline Sluggish & 0.050 & 0.702 & -0.072 & 0.496 & -0.062 & 0.636 & 0.081 & 0.533 \\
\hline Agitation & 0.138 & 0.288 & -0.031 & 0.902 & 0.167 & 0.199 & 0.030 & 0.827 \\
\hline Psychic anxiety & 0.152 & $0.24 I$ & -0.069 & 0.513 & 0.040 & 0.760 & 0.042 & 0.748 \\
\hline Somatic anxiety & 0.230 & 0.074 & -0.051 & 0.630 & 0.098 & 0.451 & 0.075 & 0.566 \\
\hline Gastrointestinal symptoms & 0.367 & $0.004^{\#}$ & 0.025 & 0.817 & 0.242 & 0.060 & 0.255 & $0.047^{*}$ \\
\hline Constitutional symptoms & $0.25 I$ & 0.051 & -0.023 & 0.827 & 0.197 & 0.128 & 0.272 & $0.034^{*}$ \\
\hline Sexual symptoms & 0.163 & 0.208 & -0.137 & 0.294 & -0.015 & 0.911 & 0.045 & 0.728 \\
\hline Hypochondriasis & 0.065 & 0.617 & -0.290 & $0.024 *$ & -0.279 & $0.030 *$ & -0.082 & 0.547 \\
\hline Loss of weight & 0.221 & 0.055 & -0.148 & 0.255 & -0.009 & 0.945 & 0.163 & 0.210 \\
\hline Self-conciousness & 0.050 & 0.703 & -0.007 & 0.955 & 0.065 & 0.621 & -0.027 & 0.839 \\
\hline Change (day and night) & -0.176 & 0.175 & -0.074 & 0.570 & -0.187 & 0.149 & -0.181 & 0.163 \\
\hline Disintegration of personality or reality & -0.063 & 0.628 & -0.150 & 0.247 & -0.156 & 0.231 & -0.077 & 0.557 \\
\hline Paranoid symptoms & 0.163 & 0.210 & -0.201 & 0.121 & -0.145 & 0.166 & -0.145 & 0.166 \\
\hline Obsessive compulsive symptoms & 0.039 & 0.703 & -0.050 & 0.703 & 0.074 & 0.571 & 0.074 & 0.575 \\
\hline Ability decline & 0.224 & 0.083 & -0.059 & 0.649 & 0.023 & 0.861 & 0.158 & 0.225 \\
\hline Hopelessness & 0.378 & $0.003^{\#}$ & -0.092 & 0.481 & 0.084 & 0.518 & 0.240 & $0.006^{\#}$ \\
\hline Inferiority & 0.030 & 0.817 & 0.012 & 0.924 & 0.027 & 0.837 & -0.113 & 0.384 \\
\hline
\end{tabular}

Notes: ${ }^{*} P<0.05 ;{ }^{*} P<0.01$.

Abbreviations: HAMD, Hamilton Depression Rating Scale; MPV, mean platelet volume; PLT, platelet; PCT, plateletcrit; PDW, platelet distribution width.

Table 4 The Correlation Analysis Between the MPVand HAMD Factor Score in the Depression Group

\begin{tabular}{|l|c|c|}
\hline \multirow{2}{*}{ HAMD } & \multicolumn{2}{|c|}{ MPV } \\
\cline { 2 - 3 } & $\mathbf{r}$ & $\boldsymbol{P}$ \\
\hline Anxiety/somatization & 0.259 & $0.044^{*}$ \\
Weight & 0.249 & 0.053 \\
Cognitive disturbance & 0.194 & 0.133 \\
Change (day and night) & -0.015 & 0.909 \\
Sluggish & 0.181 & 0.162 \\
Somnipathy & 0.064 & 0.626 \\
Hopelessness & 0.321 & $0.012^{*}$ \\
\hline
\end{tabular}

Note: $* P<0.05$.

Abbreviations: HAMD, Hamilton Depression Rating Scale; MPV, mean platelet volume.

Table 5 Comparison of CRP Level in Two Groups

\begin{tabular}{|l|c|c|c|c|}
\hline & $\mathbf{n}$ & $\mathbf{C R P}(\mathbf{m g} / \mathbf{L})$ & $\boldsymbol{Z}$ & $\boldsymbol{P}$ \\
\hline Depression group & 61 & $2.50(2.50,3.74)$ & -3.495 & $0.000^{*}$ \\
Control group & 30 & $2.50(2.50,2.50)$ & & \\
\hline
\end{tabular}

Note: $* P<0.05$.

Abbreviation: CRP, C-reactive protein. impairment in these patients, and the correlation coefficient was 0.256, $\mathrm{P}=0.046(\mathrm{P}<0.05$; Table 7).

\section{Discussion}

In this study, platelet activation and chronic inflammatory response were observed by measuring platelet parameters and CRP levels to assess the association of these factors with the clinical phenotype of depression.

First, we found that a larger proportion of patients with depression were female than male, indicating that women are at higher risk of depression; this is consistent with most existing findings. ${ }^{19}$ Second, the patients with depression in our study had a lower BMI than the healthy control subjects, as was also the case in Lawl's research. ${ }^{20-22}$ This finding, with support from the existing literature, indicates that weight loss may be an element of the inflammatory suppression link in depression. ${ }^{21}$ This could be because individuals with higher BMIs can consume more nutrients, which can eventually increase central serotonin activity and reduce depressive symptoms. ${ }^{22}$ In contrast, however, 
Table 6 The Correlation Analysis Between C-Reactive Protein (CRP) and HAMD Factor Score in the Depression Group

\begin{tabular}{|l|c|c|}
\hline \multirow{2}{*}{ HAMD } & \multicolumn{2}{|c|}{ CRP } \\
\cline { 2 - 3 } & Correlation Coefficient & $P$ \\
\hline Depressed mood & 0.038 & 0.773 \\
Guilty & 0.340 & 0.007 \\
Suicide & 0.038 & 0.773 \\
Difficulty falling asleep & 0.207 & 0.109 \\
Light sleep & 0.089 & 0.494 \\
Early awakening & -0.180 & 0.165 \\
Work and interest & 0.003 & 0.983 \\
Sluggish & -0.098 & 0.452 \\
Agitation & 0.071 & 0.586 \\
Psychic anxiety & 0.139 & 0.286 \\
Somatic anxiety & 0.194 & 0.133 \\
Gastrointestinal symptoms & 0.011 & 0.934 \\
Constitutional symptoms & 0.130 & 0.318 \\
Sexual symptoms & -0.063 & 0.631 \\
Hypochondriasis & 0.175 & 0.177 \\
Loss of weight & 0.125 & 0.336 \\
Self-conciousness & -0.017 & 0.897 \\
Change (day and night) & 0.052 & 0.689 \\
Disintegration of personality & 0.180 & 0.166 \\
or reality & & \\
Paranoid symptoms & 0.126 & 0.333 \\
Obsessive compulsive & 0.015 & 0.909 \\
symptoms & 0.162 & 0.213 \\
Ability decline & 0.136 & 0.298 \\
Hopelessness & & \\
Inferiority & & \\
\hline Note: & & \\
\hline
\end{tabular}

Note: $* P<0.05$.

Abbreviations: HAMD, Hamilton Depression Rating Scale; CRP, C-reactive protein.

Table 7 The Correlation Analysis Between C-Reactive Protein and HAMD Factor Score in the Depression Group

\begin{tabular}{|l|c|c|}
\hline \multirow{2}{*}{ HAMD } & \multicolumn{2}{|c|}{ CRP } \\
\cline { 2 - 3 } & Correlation Coefficient & $P$ \\
\hline Anxiety/somatization & 0.191 & 0.140 \\
Weight & 0.150 & 0.249 \\
Cognitive disturbance & 0.256 & $0.046^{*}$ \\
Change (day and night) & 0.098 & 0.453 \\
Sluggish & -0.010 & 0.937 \\
Somnipathy & 0.005 & 0.971 \\
Hopelessness & 0.203 & 0.116 \\
\hline
\end{tabular}

Note: ${ }^{*} P<0.05$.

Abbreviations: HAMD, Hamilton Depression Rating Scale; CRP, C-reactive protein.

one study found that patients with atypical depression had higher BMI than control subjects. ${ }^{23}$

In the current study, the MPV levels in the severe depression group (HAMD > 35) were higher than those in the mild and moderate depression groups and the healthy control subjects, indicating that MPV is increased in patients with severe depression. Cai et al's study, ${ }^{24}$ which compared 103 patients with MDD and 106 healthy subjects, showed elevated MPV in the MDD group compared with the healthy controls, with no significant difference between the PLT values for the two groups. These results are consistent with the idea that elevated MPV mainly reflects platelet activation and the chronic inflammatory response in vivo. Considering the results of the current study, this could lead to the significantly increased platelet activation and chronic inflammatory response in MDD. With an increase of MPV, platelets are further activated to release 5-HT into the serum, causing a relative increase of 5-HT in the serum. This is consistent with previous studies showing a decrease of 5-HT in platelets and the neurosynaptic space in patients with depression. ${ }^{25}$ In the current study, we also found that, in patients with depression, MPV was positively correlated with HAMD-scale scores for gastrointestinal symptoms, work and interest, despair, and anxiety/somatization factors. Recent gastrointestinal studies have suggested a positive association of MPV with cirrhosis, gastric ulcers, and chronic gastritis with Helicobacter pylori infection, ${ }^{26-28}$ indicating that an increase in MPV may result from the enhancement of chronic inflammation. Although there have been no reports of MPV treatment in patients with MDD, some scholars have proposed that anti-inflammatory treatment can have a good effect on depression. ${ }^{29}$

In the current study, PDW in patients with depression was found to be positively associated with gastrointestinal and constitutional symptoms, as well as hopelessness, in the HAMD scale. This is similar to this correlation study of MPV with each scale. Previous studies have also noted elevated PDW and PCT in patients with inflammatory bowel disease compared with healthy control subjects. ${ }^{30}$ Additionally, Yang et al ${ }^{31}$ showed that patients with depression had increased MPV and PDW compared with control subjects, suggesting the possible mechanism of these parameters enhancing platelet activity, eventually leading to depression. Patients with depression are prone to systemic symptoms, despair, somatic anxiety, and various other symptoms. However, there is a lack of direct clinical and animal studies concerning PDW and systemic symptoms, despair, and somatic anxiety; therefore, the relationships between PDW and these factors require further investigation. Our 
findings also indicated negative correlations of PLT and PCT with the suspected illness and poor sleep factors of the HAMD scale in patients with depression. No study has directly pointed out a correlation of PLT and PCT with insomnia and disease suspicion; however, some researchers have found that PLT is significantly reduced in patients with obstructive sleep apnea syndrome, while MPV and PDW increase. ${ }^{32}$ As PCT is the product of MPV and PLT, and PCT changes are generally consistent with PLT changes, ${ }^{33}$ it can be speculated that PCT is negatively associated with poor sleep.

No significant differences in PDW, PLT, or PCT results for the different groups were found in the current study; the findings confirmed only elevated MPV in patients with severe depression compared with the control subjects. It is likely that, in cases of severe depression, the abilities of MPV to activate platelets and respond to chronic inflammation are strong, while the other three parameters may respond more slowly in patients with depression. Additional large clinical sample studies are required for clarity on the association of these indicators with depression.

This study found elevated CRP in patients with depression compared with healthy control subjects, which is consistent with findings from large samples in clinical studies. $^{34,35}$ Our findings indicated a positive association of CRP content with the guilt and cognitive disturbance factor scores in the HAMD scale for patients with depression; as CRP content increased in these patients, their guilt and awareness impairment factor scores also increased. The HAMD recognition disorder factor score encompasses guilt, shock, and paranoid symptoms, so an increased sense of guilt may also increase the recognition disorder factor score. $\mathrm{Hu}$ et $\mathrm{al}^{36}$ reported a positive correlation between patients with depression and the mental anxiety, guilt, and shock scores in the HAMD scale, which is consistent with the results of the current study.

A clinical study by Köhler showed that combining selective serotonin reuptake inhibitors with antiinflammatory agents resulted in improved treatment effects for depression. ${ }^{37}$ However, research on such medication combinations is still relatively limited, and we hope to provide new ideas for treatments using anti-inflammatory and antidepressant drugs in the future.

The present study had some limitations. First, the sample size was small; future research should include larger sample sizes and multicenter clinical studies. Second, for the classification of depressive symptoms in future studies, it is necessary to control for confounders that could potentially interfere with the immunodepression relationship. This will allow for further exploration of the physiopathological mechanisms linking CRP, classical platelet parameters, and elements of the clinical phenotype of depression (gastrointestinal tract, sleep, disease suspicion, etc.) and for identification of the cellular metabolic pathways that mediate these changes.

\section{Conclusion}

Classical platelet parameters (PLT, MPV, PCT, PDW) and CRP were shown to be associated with specific depressive symptoms and cognitive impairment factors, including sleep, gastrointestinal symptoms, hypochondriasis, losing interest in work, and despair. These results suggest that both platelet parameters and CRP could be suitable biomarkers for predicting the occurrence and prognosis of depression, thus providing a new target for its treatment.

\section{Acknowledgments}

1) We thank Fujian Commission of Health and Family Planning and Fujian University of Traditional Chinese Medicine for their financial support. 2) We thank Dr. Dengzi Zheng, Deputy Chief Physician of the Inspection Department of Fujian Second People's Hospital, for his analysis of the blood cells in this work. 3) We thank Xiao-Qian Wei, Jia-Wen Li, MingYang Chen and other graduate students of Fujian University of Traditional Chinese Medicine, for their great help in finding literature.

\section{Funding}

This work was supported by Fujian Provincial Health and Family Planning Commission (No.zlcxn09) and Fujian University of Traditional Chinese Medicine (No. X2019012).

\section{Disclosure}

The authors declare that they have no competing interests.

\section{References}

1. Murray CJ, Lopez AD. Alternative projections of mortality and disability by cause 1990-2020: Global Burden of Disease Study. Lancet. 1997;349:1498-1504. doi:10.1016/S0140-6736(96)07492-2

2. Beurel E, Toups M, Nemeroff CB. The bidirectional relationship of depression and inflammation: double trouble. Neuron. 2020;10 7:234-256. doi:10.1016/j.neuron.2020.06.002

3. Adams TB, Wharton CM, Quilter L, Hirsch T. The association between mental health and acute infectious illness among a national sample of 18- to 24-year-old college students. J Am Coll Health. 2008;56:657-663. doi:10.3200/JACH.56.6.657-664 
4. Yirmiya R, Rimmerman N, Reshef R. Depression as a microglial disease. Trends Neurosci. 2015;38:637-658. doi:10.1016/j.tins.2015. 08.001

5. Kokacya MH, Copoglu US, Kivrak Y, Ari M, Sahpolat M, Ulutas KT. Increased mean platelet volume in patients with panic disorder. Neuropsychiatr Dis Treat. 2015;11:2629-2633. doi:10.2147/NDT.S94147

6. Lu WB, Shen GF, Li Y, Gan LY. [Assessment of the changes in platelet in the treatment of depression by duloxetine]. Chin J Drug Abus Prev. 2015;21:336-338. Chinese.

7. Chen DN, Wang YM. [Effect of sertraline hydrochloride combined with aspirin on suicide risk in patients with depression]. Chongqing Med. 2018;47:4379-4382, 4386. Chinese.

8. Tang WC, Li M, Yi HY, Luo FF, Yang L. [Influence of aspirin for the expression of cytokines in hippocampus of chronic stress depression rats]. Sichuan Ment Health. 2018;31:313-317. Chinese.

9. Canan F, Dikici S, Kutlucan A, et al. Association of mean platelet volume with DSM-IV major depression in a large community-based population: the MELEN study. J Psychiatr Res. 2012;46:298-302. doi:10.1016/j.jpsychires.2011.11.016

10. Wang RT, Jin D, Li Y, Liang QC. Decreased mean platelet volume and platelet distribution width are associated with mild cognitive impairment and Alzheimer's disease. J Psychiatr Res. 2013;47: 644-649. doi:10.1016/j.jpsychires.2013.01.014

11. Liang QC, Jin D, Li Y, Wang RT. Mean platelet volume and platelet distribution width in vascular dementia and Alzheimer's disease. Platelets. 2014;25:433-438. doi:10.3109/09537104.2013.831064

12. Chen $\mathrm{SH}, \mathrm{Bu} \mathrm{XL}$, Jin WS, et al. Altered peripheral profile of blood cells in Alzheimer disease: a hospital-based case-control study. Medicine. 2017;96:e6843. doi:10.1097/MD.0000000000006843

13. Gialluisi A, Bonaccio M, Di Castelnuovo A, et al.; Moli-Sani Study Investigators. Lifestyle and biological factors influence the relationship between mental health and low-grade inflammation. Brain Behav Immun. 2020;85:4-13. doi:10.1016/j.bbi.2019.04.041

14. Gialluisi A, Izzi B, Di Castelnuovo A, et al. Revisiting the link between platelets and depression through genetic epidemiology: new insights from platelet distribution width. Haematologica. 2020;105:e246-e248. doi:10.3324/haematol.2019.222513

15. Wang ZZ, Chen NH. [Depression and inflammation]. Acta Neuropharmacologica. 2013;3:27-37. Chinese.

16. Miller GE, Freedland KE, Duntley S, Carney RM. Relation of depressive symptoms to C-reactive protein and pathogen burden (cytomegalovirus, herpes simplex virus, Epstein-Barr virus) in patients with earlier acute coronary syndromes. Am J Cardiol. 2005 Feb 1;95(3):317-21. doi:10.1016/j.amjcard.2004.09.026. PMID: 15670537.

17. Chu X, Chen Q, Dong H, Xu J, Yu K. [Study of the relationship between hypersensitivity C-responsive protein and tumor necrosis factor $\alpha$ expression in depressed patients]. $J$ Psychiatry. 2011;24:457-458. Chinese.

18. Liu Z, Chen JJ, Zhou J, Xie P. [Association of blood C-reactive protein with depression: a systematic review]. J Chongqing Med Uni. 2015;40:60-65. Chinese.

19. Zhao L, Han G, Zhao Y, et al. Gender differences in depression: evidence from genetics. Front Genet. 2020;11:562316. doi:10.3389/ fgene.2020.562316

20. Lawlor DA, Hart CL, Hole DJ, Gunnell D, Davey Smith G. Body mass index in middle life and future risk of hospital admission for psychoses or depression: findings from the Renfrew/Paisley study. Psychol Med. 2007;37:1151-1161. doi:10.1017/S0033291707000384
21. Qin T, Liu W, Yin M, et al. Body mass index moderates the relationship between C-reactive protein and depressive symptoms: evidence from the China Health and Retirement Longitudinal Study. Sci Rep. 2017;7:39940. doi:10.1038/srep39940

22. Zhang L, Liu K, Li H, et al. Relationship between body mass index and depressive symptoms: the "fat and jolly" hypothesis for the middle-aged and elderly in China. BMC Public Health. 2016;16:1201. doi:10.1186/s12889-016-3864-5

23. Lamers F, Vogelzangs N, Merikangas KR, de Jonge P, Beekman AT, Penninx BW. Evidence for a differential role of HPA-axis function, inflammation and metabolic syndrome in melancholic versus atypical depression. Mol Psychiatry. 2013;18:692-699. doi:10.1038/mp.2012.144

24. Cai LQ, Xu LY, Wei LL, Chen W. [Relaitonship of mean platelet volume to MDD: a retrospecitve study]. Shanghai Arch Psychiat. 2017;19 (1):21-29. Chinese.

25. Yang XR, Song XP, Zeng CB, Yang XM. [Physiological characteristics and detecting methods of 5-HT in brain]. Sichuan Ment Health. 2017;30:196-200. Chinese.

26. Zeng TT, Tan LM, Tian YJ, et al. [Predictive and diagnostic value of red blood cell distribution width and mean platelet volume in autoimmune hepatitis related cirrhosis]. Med J Chin People's Liberation Army. 2018;43:1039-1043. Chinese.

27. Chen XY, Ji ML, Liu XH, et al. [Differential diagnosis of gastric cancer and gastric ulcer by joint detection of peripheral blood MPV, PDW and serum CEA]. J Mod Lab Med. 2019;34:107-110, 141. Chinese.

28. Umit H, Umit EG. Helicobacter pylori and mean platelet volume: a relation way before immune thrombocytopenia? Eur Rev Med Pharmacol Sci. 2015;19:2818-2823.

29. Akhondzadeh S, Jafari S, Raisi F, et al. Clinical trial of adjunctive celecoxib treatment in patients with major depression: a double blind and placebo controlled trial. Depress Anxiety. 2009;26:607-611. doi: $10.1002 /$ da.20589

30. Öztürk ZA, Dag MS, Kuyumcu ME, et al. Could platelet indices be new biomarkers for inflammatory bowel diseases? Eur Rev Med Pharmacol Sci. 2013;17:334-341.

31. Yang JX, Wufuer A, Maimaitiming G. [Relationship between depression and change of platelet activity in elderly patients with coronary heart disease]. Chin Heart J. 2014;26:465-467. Chinese.

32. Han SZ, Sun JJ, Ping F, Yang YH, Zhao JC. [Changes in platelet parameters and their clinical significance in patients with obstructive sleep apnea and low-channel syndrome]. Hebei Med J. 2006;28: 28-29. Chinese.

33. Liu HX, Chen M. [Analysis of the correlation of platelet parameter with ageing]. J Taishan Med Coll. 2010;31:280-281. Chinese.

34. Wysokiński A, Margulska A, Strzelecki D, Kłoszewska I. Levels of C-reactive protein (CRP) in patients with schizophrenia, unipolar depression and bipolar disorder. Nord J Psychiatry. 2015;69: 346-353. doi:10.3109/08039488.2014.984755

35. Haapakoski R, Mathieu J, Ebmeier KP, Alenius H, Kivimäki M. Cumulative meta-analysis of interleukins 6 and $1 \beta$, tumour necrosis factor $\alpha$ and C-reactive protein in patients with major depressive disorder. Brain Behav Immun. 2015;49:206-215. doi:10.1016/j.bbi.2015.06.001

36. Hu W, Zhang CY, Wei YF, Zhao HF, Geng DQ. [Study of serum hypersensitive C-reactive protein level in first-episode patients with retarded depression]. J Clin Psychiatry. 2015;25:251-252. Chinese.

37. Köhler-Forsberg O, N Lydholm C, Hjorthøj C, Nordentoft M, Mors O, Benros ME. Efficacy of anti-inflammatory treatment on major depressive disorder or depressive symptoms: meta-analysis of clinical trials. Acta Psychiatr Scand. 2019;139:404 419. doi:10.1111/acps.13016 


\section{Publish your work in this journal}

The International Journal of General Medicine is an international, peer-reviewed open-access journal that focuses on general and internal medicine, pathogenesis, epidemiology, diagnosis, monitoring and treatment protocols. The journal is characterized by the rapid reporting of reviews, original research and clinical studie across all disease areas. The manuscript management system is completely online and includes a very quick and fair peer-review system, which is all easy to use. Visit http://www.dovepress.com/ testimonials.php to read real quotes from published authors.

Submit your manuscript here: https://www.dovepress.com/international-journal-of-general-medicine-journal 